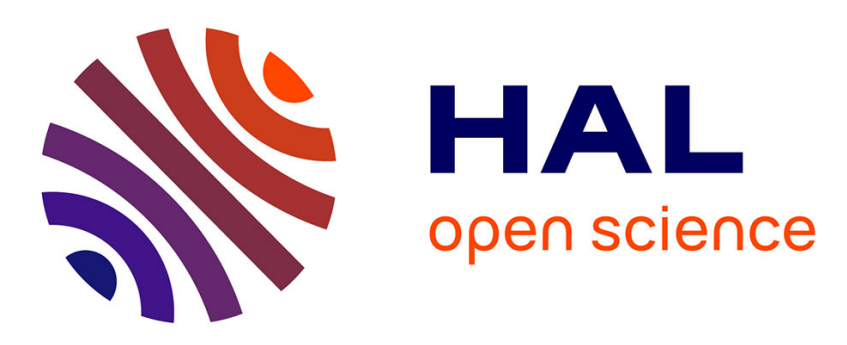

\title{
Few-Molecule Strong Coupling with Dimers of Plasmonic Nanoparticles Assembled on DNA
}

Jeanne Heintz, Nemanja Markešević, Elise y Gayet, Nicolas Bonod, Sébastien Bidault

\section{- To cite this version: \\ Jeanne Heintz, Nemanja Markešević, Elise y Gayet, Nicolas Bonod, Sébastien Bidault. Few-Molecule Strong Coupling with Dimers of Plasmonic Nanoparticles Assembled on DNA. ACS Nano, 2021, 15 (9), pp.14732-14743. 10.1021/acsnano.1c04552 . hal-03364660}

\author{
HAL Id: hal-03364660 \\ https://hal.science/hal-03364660
}

Submitted on 4 Oct 2021

HAL is a multi-disciplinary open access archive for the deposit and dissemination of scientific research documents, whether they are published or not. The documents may come from teaching and research institutions in France or abroad, or from public or private research centers.
L'archive ouverte pluridisciplinaire HAL, est destinée au dépôt et à la diffusion de documents scientifiques de niveau recherche, publiés ou non, émanant des établissements d'enseignement et de recherche français ou étrangers, des laboratoires publics ou privés. 


\section{Few-Molecule Strong-Coupling with Dimers of Plasmonic Nanoparticles Assembled on DNA}

Jeanne Heintz, ${ }^{\dagger}$ Nemanja Markešević, ${ }^{\dagger}, \Phi^{-}$Elise Y. Gayet, ${ }^{\dagger}$ Nicolas Bonod ${ }^{\ddagger}$ and Sébastien Bidault ${ }^{*} \dagger$

$\dagger$ Institut Langevin, ESPCI Paris, Université PSL, CNRS, 1 rue Jussieu, 75005 Paris, France

$\ddagger$ Aix Marseille Univ, CNRS, Centrale Marseille, Institut Fresnel, 13013 Marseille, France ICurrent address: Department of Physics and Nanoscience Center, University of Jyväskylä, P.O. Box 35, FI-40014 Jyväskylä, Finland

E-mail: sebastien.bidault@espci.fr 


\begin{abstract}
Hybrid nanostructures, in which a known number of quantum emitters are strongly coupled to a plasmonic resonator, should feature optical properties at room temperature such as few-photon nonlinearities or coherent superradiant emission. We demonstrate here that this coupling regime can only be reached with dimers of gold nanoparticles in stringent experimental conditions, when the interparticle spacing falls below $2 \mathrm{~nm}$. Using a short transverse DNA double-strand, we introduce 5 dye molecules in the gap between two $40 \mathrm{~nm}$ gold particles and actively decrease its length down to sub-2 nm values by screening electrostatic repulsion between the particles at high ionic strengths. Single-nanostructure scattering spectroscopy then evidences the observation of a strong-coupling regime in excellent agreement with electrodynamic simulations. Furthermore, we highlight the influence of the planar facets of polycrystalline gold nanoparticles on the probability of observing strongly coupled hybrid nanostructures.
\end{abstract}

Keywords: strong coupling, plasmonics, DNA nanotechnology, self-assembly, scattering spectroscopy, dye molecules 
At room temperature, light-matter interactions in condensed media are fundamentally limited by electron-phonon coupling. For instance, while the excitation cross-section of an isolated atom, or of a single quantum emitter at cryogenic temperatures, can reach the electric-dipolar unitary limit (about one half of the wavelength squared, meaning that more than $50 \%$ of incoming photons should interact for a diffraction-limited excitation); ${ }^{1,2}$ this value is reduced by 6-7 orders of magnitude for a fluorescent molecule or for a colloidal quantum dot at room temperature because of homogeneous phonon broadening. ${ }^{3}$ In order to render the optical properties of single quantum emitters (such as single-photon emission and nonlinearities $)^{4-8}$ efficiently accessible at room temperature and in condensed media, it is essential to enhance and optimize these interaction cross-sections.

One solution is to strongly couple quantum emitters to an optical cavity that exhibits a large optical interaction cross-section, leading to the appearance of hybridized polaritonic eigenmodes. ${ }^{9,10}$ With $N$ emitters, coherent many-body effects should arise such as superradiance, ${ }^{9,11}$ but a strong coupling regime also provides degrees of freedom to actively tune photophysical and chemical processes such as energy transfer ${ }^{12-14}$ or reactivity landscapes. ${ }^{15-17}$ In the case of a single quantum emitter, it should allow optical properties, such as singlephoton nonlinearities and ultrafast switching, to be accessible at room temperature. ${ }^{4,5,7,8}$ Single-emitter strong coupling in microcavities has been demonstrated in a controlled and reproducible way at cryogenic temperatures. ${ }^{4,7,8,18}$ However, homogeneously-broadened emitters at room temperature can only efficiently couple to low-quality resonators that must therefore confine and enhance the electromagnetic field in deeply sub-wavelength volumes to preserve strong Purcell factors. ${ }^{19}$ This is achievable with plasmonic resonators, especially when strongly coupled to a large number of emitters with strong oscillator strengths such as $J$-aggregates. ${ }^{9,10,20-23}$ Room-temperature single-molecule strong coupling was also demonstrated $^{24}$ in self-assembled resonators, ${ }^{25-27}$ in top-down fabricated nanocavities ${ }^{28}$ or using scanning-probe microscopy. ${ }^{29}$

Resonators obtained using self-assembly and lithography have the strong advantage of being 
scalable but do not allow an active modulation of the position of the emitter in the resonator and, in general, the number of emitters is not known a priori, ${ }^{25,26,28}$ or the coupling strength is below the visibility criterion for strong coupling. ${ }^{27}$ On the other hand, in the case of scanning probe microscopy, it is possible to directly control the position of one emitter with respect to the resonator at the nanoscale ${ }^{29}$ but this approach is not scalable.

We demonstrate here that DNA-based self-assembly ${ }^{30-32}$ provides an active control over both the number of emitters and their position, at the nanoscale, in a resonant dimer of gold nanoparticles (AuNPs). We develop a robust self-assembly strategy that exploits a single 50bp DNA double-strand and an active modulation of the local ionic strength in order to produce $40 \mathrm{~nm}$ AuNP dimers with sub-2 nm gaps in $80 \%$ of the sample, as experimentally verified by single-nanostructure scattering spectroscopy. By introducing 5 ATTO647N molecules on the DNA strand, we demonstrate that hybrid strongly-coupled modes are only observed in the scattering spectra of single dimers when the interparticle distance decreases below $2 \mathrm{~nm}$. From the spectral positions of the measured hybrid modes, we can infer coupling strengths ranging between $50 \mathrm{meV}$ and $150 \mathrm{meV}$, in excellent agreement with theoretical calculations. Furthermore, electrodynamic simulations suggest that the low probability with which strong coupling is observed, is due to the planar facets of the employed polycrystalline $40 \mathrm{~nm}$ AuNPs. These results thus evidence how few-molecule strong coupling can be achieved in plasmonic resonators with an active control of the number of emitters and of the interparticle distance thanks to DNA-based self-assembly, and which parameters should be optimized to reach this regime in a reproducible way. 


\section{Results and Discussion}

\section{Theoretical investigation of visible strong coupling in dimers of gold nanoparticles}

The DNA-based assembly of dimers of plasmonic nanoparticles, with diameters ranging from $40 \mathrm{~nm}$ and $100 \mathrm{~nm}$, has been shown to be a flexible and robust approach to enhance the spontaneous emission of single emitters in a weak coupling regime. ${ }^{19,33-40}$ In particular, it allows a nanoscale control over the interparticle distance and over the position of the emitter in the gap. ${ }^{19,34,36,38}$ This method also permits the controlled introduction of multiple emitters, in particular to modulate resonant energy transfer. ${ }^{41-43}$ It is therefore an attractive approach to efficiently couple a known number of fluorescent molecules to a plasmonic resonator in order to reach a strong coupling regime.

To investigate whether single-molecule strong coupling is achievable in dimers of gold nanoparticles, we use electrodynamic simulations to estimate the emitter-resonator coupling strength $g$, which is defined as: ${ }^{44}$

$$
g=\frac{\sqrt{F_{p} \gamma_{p} \Gamma_{R 0}}}{2}
$$

with $F_{p}$ the Purcell factor of the resonator for a given position and orientation of the emitter, $\gamma_{p}$ the decay rate of the resonator, and $\Gamma_{R 0}$ the radiative decay rate of the emitter without the resonator. The Purcell factor $F_{p}$ is computed as the change in the local density of optical states (LDOS) for the emitter, with a given position and orientation in the resonator, relative to a homogeneous medium without the resonator. ${ }^{44}$ This is done by substituting the quantum emitter for a classical electric dipole and estimating the total power that the latter dissipates when coupled to the resonator $P_{t o t}$, compared to the power radiated without the

resonator $P_{R 0}$, giving $F_{p}=P_{t o t} / P_{R 0} \cdot{ }^{37,45}$ It is important to note that this computed Purcell factor is emitter-independent as it does not take into account the initial quantum yield $\Phi_{0}$ of the fluorescent molecule. It is possible to infer a relative change of the LDOS that takes $\Phi_{0}$ 
into account and then substitute $\Gamma_{0}$ for $\Gamma_{R 0}$ in equation 1, giving the same estimated value for the coupling strength $g$. By estimating the Purcell factor with a purely radiative classical dipole, the advantage is that changing the emitter will modify $\Gamma_{R 0}$ but not the computed value of $F_{p}$.

It is also possible to estimate the radiative yield $\Phi$ of the resonator, for a given position and orientation of the dipolar emitter, by computing the total power radiated by the classical dipole $P_{R}$ with the resonator, namely $\Phi=P_{R} / P_{\text {tot }}$. As spontaneous emission from a coupled molecule-plasmonic resonator system is dominated by the resonator, this radiation efficiency is a good estimate of the final emission quantum yield. ${ }^{33,37}$

By approximating the gold nanoparticles as gold nanospheres (AuNSs), it is then possible to compute the Purcell factor $F_{p}$ and the radiative yield $\Phi$ for plasmonic dimers as a function of the interparticle spacing using Mie theory ${ }^{46}$ or the Boundary Element Method (BEM $)^{47}$ and for three particle diameters $(40 \mathrm{~nm}, 60 \mathrm{~nm}$ and $80 \mathrm{~nm}$ ), as shown in Figure 1-a. In this case, the dipolar emitter is positioned in the center of the dimer and oriented longitudinally, i.e. along the dimer axis. Furthermore, in Figure 1, we consider the maximum values of $F_{p}$ and $g$ obtained when light radiated by the dipole matches spectrally the longitudinal mode of the cavity $\left(\omega_{p}\right)$. The dielectric constant of gold is taken from tabulated data, ${ }^{48}$ while the refractive index of the environment is chosen at 1.4 to be compatible with experiments performed on DNA-templated gold particle dimers. ${ }^{19,37,49}$ Since recent reports have shown that classical electrodynamics simulations remain valid down to $1 \mathrm{~nm}$ gaps, but that quantum tunneling significantly influences plasmon coupling for smaller spacings, ${ }^{50,51}$ we do not perform calculations for sub-1 nm spacings.

Figure 1-a shows that the LDOS is significantly increased in the center of the dimer when the spacing $s$ is decreased, reaching values of $F_{p}$ exceeding $10^{5}$ for all particle diameters when $s$ is below $2 \mathrm{~nm}$. For all interparticle distances, $F_{p}$ is larger for $40 \mathrm{~nm}$ particles as they locally feature larger curvatures at the position of the emitter than $60 \mathrm{~nm}$ or $80 \mathrm{~nm}$ AuNSs. However, since smaller AuNSs feature lower scattering cross-sections, the radiative 

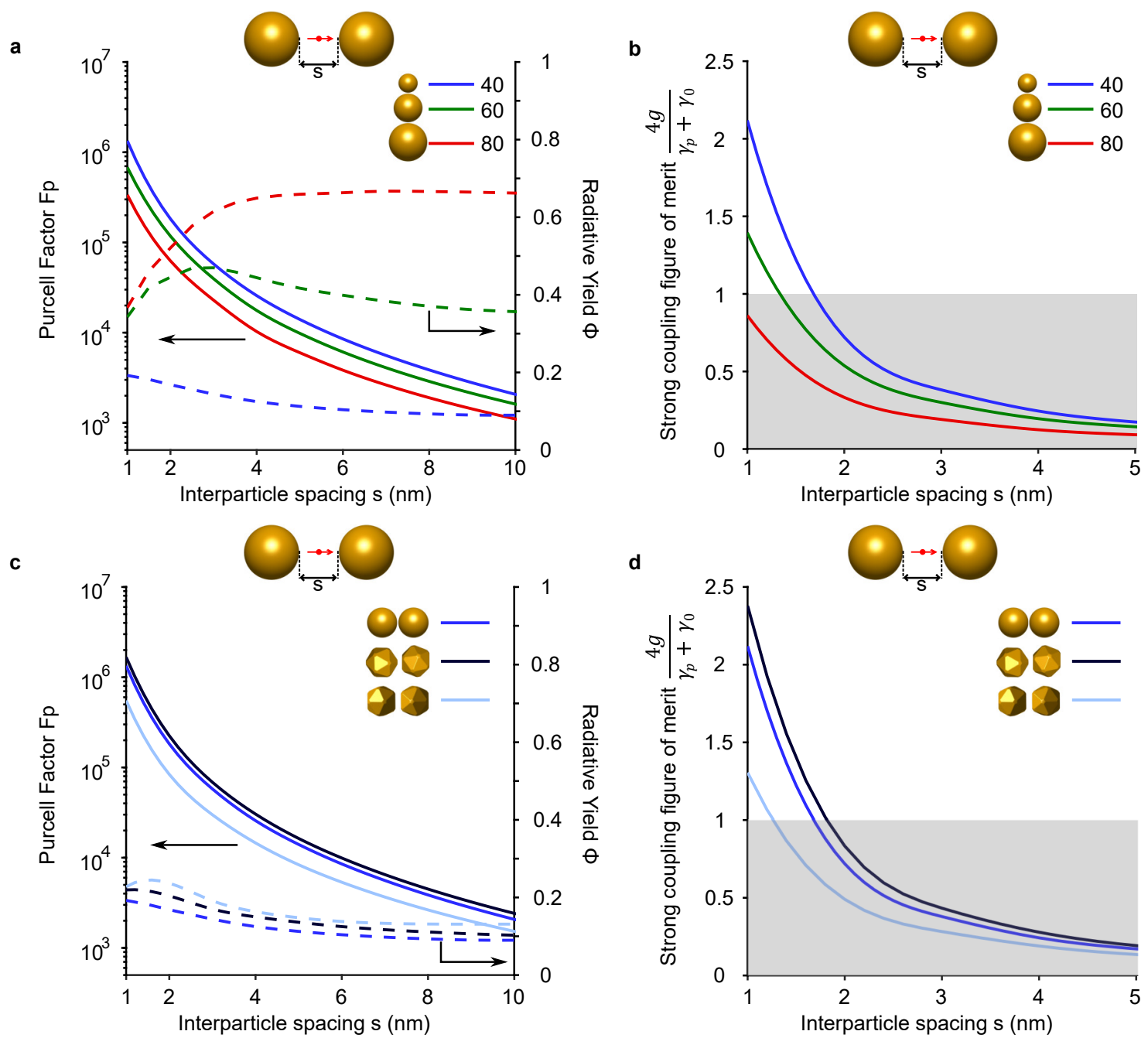

Figure 1: Theoretical investigation of strong coupling in dimers of gold nanoparticles. (a) Computed relative change of the local density of optical states, or Purcell factor $F_{p}$ (solid lines), and radiative yield $\Phi$ (dashed lines), in dimers of $40 \mathrm{~nm}$ (blue data), $60 \mathrm{~nm}$ (green) and $80 \mathrm{~nm}$ (red) diameter gold nanospheres for a longitudinally coupled electric dipole positioned in the center and as a function of the interparticle spacing $s$. For all values of $s$, the emission wavelength of the dipole matches the resonance wavelength of the AuNS dimer. (b) Visible strong coupling figure of merit $4 g /\left(\gamma_{p}+\gamma_{0}\right)$ (solid line, see text for details) as a function of $s$ in the same geometry as (a). (c) Computed Purcell factor $F_{p}$ (solid lines), and radiative yield $\Phi$ (dashed lines), in dimers of $40 \mathrm{~nm}$ AuNPs for a longitudinally coupled emitter positioned in the center and as a function of the interparticle spacing $s$ : perfect spheres (blue data) and rounded icosahedra, assembled with parallel facets (light blue) or with a tip facing a planar facet (dark blue). All particle volumes are identical. (d) Visible strong coupling figure of merit $4 g /\left(\gamma_{p}+\gamma_{0}\right)$ (solid line) as a function of $s$ in the same geometry as (c).

yield $\Phi$ follows the opposite trend: it is systematically larger for bigger spheres. We observe a slight increase of the quantum yield when the resonance wavelength $\omega_{p}$ corresponds to 
the minimum of the imaginary part of the dielectric constant of gold: for instance, for 60 $\mathrm{nm}$ AuNS dimers with a spacing around $3 \mathrm{~nm}$ or $40 \mathrm{~nm}$ AuNS dimers with $s=1 \mathrm{~nm}$. In practice, larger radiative damping in the resonator will increase $\gamma_{p}$ : while this increases $g$, it also reduces the visible energy splitting of hybrid modes in strong coupling by increasing their spectral width. ${ }^{25,27}$

To estimate whether the computed LDOS increase is sufficient for the emitter-resonator system to feature strongly-coupled hybrid modes, we consider a strong coupling figure of merit which corresponds to a visibility criterion: the energy splitting $\Omega$ between the modes should exceed the sum of half the linewidths of the initial uncoupled modes $\left(\frac{\gamma_{p}}{2}+\frac{\gamma_{0}}{2}\right.$, with $\gamma_{0}$ the dephasing rate of the emitter). ${ }^{9,27}$ In practice, we compute the total damping rate of the resonator $\gamma_{p}$ and consider that the electronic resonance of the emitter $\omega_{0}$ matches the plasmonic resonance $\omega_{p}=\omega_{0}$, meaning that $\Omega=2 g \cdot^{9,27}$ In this case, the visibility criterion simplifies as:

$$
\frac{4 g}{\gamma_{p}+\gamma_{0}}>1
$$

For typical fluorescent dyes, the radiative lifetime $\tau_{R 0}$ ranges between 2 ns and 7 ns: we thus consider an initial decay rate $\Gamma_{R 0}=0.2 \mu \mathrm{eV}\left(=\hbar / \tau_{R 0}\right.$, with $\hbar$ the reduced Planck constant $)^{27}$ to estimate $g$ from the computed values of $F_{p}$ and $\gamma_{p}$. In the literature, a wide range of values were used for the dephasing rate $\gamma_{0}$ of the emitter, ranging from $25 \mathrm{meV}$ to 160 meV..$^{9,24,25,44}$ Furthermore, recent measurements on single molecules indicate typical values of $\gamma_{0}$ around $20 \mathrm{meV} .{ }^{52}$ We therefore chose a conservative value of $\gamma_{0}=50 \mathrm{meV}$ to infer the strong coupling figure of merit as a function of the interparticle spacing in Figure 1-b.

We observe that, for a longitudinally-coupled emitter, single-molecule strong coupling should be visible for $40 \mathrm{~nm}$ AuNS dimers when the spacing is below $2 \mathrm{~nm}$, while the visibility figure of merit is only larger than 1 for $s=1 \mathrm{~nm}$ with $60 \mathrm{~nm}$ particles and remains below 1 with $80 \mathrm{~nm}$ AuNSs. These simulations therefore demonstrate that visible strong coupling with a single emitter is particularly difficult to achieve in dimers of gold nanospheres. Furthermore, these calculations show that it is essential to minimize the damping rate $\gamma_{p}$ of the resonator by 
choosing smaller particles. However, this reduction is done by lowering radiative damping at the cost of the radiative yield $\Phi$. In practice, with $40 \mathrm{~nm}$ particles, strong-coupling is achievable but the coupled system will feature a very weak emission quantum yield. This means that scattering spectroscopy is then better suited than photoluminescence measurements to analyze such hybrid nanostructures. We did not consider smaller spheres to further increase $F_{p}$ because their scattering cross-section would become too small for single-nanostructure spectroscopy in darkfield microscopy. ${ }^{49,53}$

Figures 1-a-b only consider a longitudinally coupled dipolar emitter, positioned exactly in the center of the dimer. However, DNA-based self-assembly does not allow a direct control over the orientation of the emitter and other orientations of its transition dipole in plasmonic resonators will reduce the coupling strength. ${ }^{19,27,35-37}$ In practice, the coupling criterion is reduced by a factor of 2 when the emitter takes an orientationally averaged value of $\pi / 3$ and barely remains above 1 for $s=1 \mathrm{~nm}$ (see Figure Figure S1-a of the Supporting Information), evidencing that the probability of observing single-molecule strong coupling will be weak, even at extreme nanoscale spacings. Furthermore, these simulations indicate that strongcoupling will never be observed for transversely oriented emitters.

Since these calculations indicate that reaching single-molecule strong coupling in dimers of nanospheres is experimentally challenging, it is interesting to consider whether it is possible to include more than one emitter in the gap by computing the visible strong coupling figure of merit as a function of the transverse position of the emitter in the gap. For longitudinally coupled-emitters in a $1 \mathrm{~nm}$ interparticle spacing, strong coupling should remain visible when the emitter is $\pm 3.5 \mathrm{~nm}$ away from the dimer axis (see Figure S1-b). This leaves room for several emitters to be positioned in the gap in order to couple coherently with the plasmonic resonator. In the approximation that $N$ emitters have the same coupling strength $g$ with the resonator, the total coupling strength is then multiplied by $\sqrt{N} \cdot{ }^{9,10,22,44}$ To avoid coherent coupling between emitters, a relative distance of at least $1 \mathrm{~nm}$ should however be maintained. ${ }^{21}$ 
It is important to note that Mie theory calculations and BEM simulations agree quantitatively, demonstrating that BEM simulations are reliable even for extreme nanoscale gaps between plasmonic particles and can thus be applied to the study of anisotropic particles (for a $1 \mathrm{~nm}$ interparticle spacing, discrepancies remain below $5 \%$ as shown in Figure S1 of the Supporting Information). It is therefore possible to analyze the influence of the nanoparticle morphology if it deviates from a perfect sphere. Indeed, polycrystalline gold particles typically feature faceted shapes that strongly influence plasmonic resonances. ${ }^{54,55}$ It was actually proposed that nanoscale facets, and the associated tips, have a strong influence on the experimental observation of single-molecule strong coupling in self-assembled plasmonic resonators. ${ }^{26}$ Indeed, high local curvatures, associated with a lightning-rod effect, ${ }^{56}$ should increase the coupling strength $g$.

Faceted polycrystalline gold nanoparticles can be readily approximated as rounded icosahedra in order to study the influence of nanoscale facets. ${ }^{55}$ Figures 1-c-d show the evolution of the computed values of $F_{p}, \Phi$ and of the strong-coupling figure of merit as a function of the particle shape, while considering the same volume of a $40 \mathrm{~nm}$ sphere. We consider two extreme relative orientations for the rounded icosahedra: when the AuNPs assemble by two planar facets or with a tip facing a planar facet. In the case of DNA-templated dimers of colloidal particles, assembly by the tips has a very low probability of occurring as, at short spacings, plasmonic particles assemble nearly systematically on their larger facets. ${ }^{57}$.

As expected, the highest expected Purcell factor and coupling figure of merit are observed when one tip of the faceted nanoparticle is introduced in the gap in order to take advantage of the lightning rod effect. The gain with respect to an ideal sphere is limited to $\sim 10 \%$ because we considered a realistic local radius of curvature for the AuNP tip. In the more probable case of an assembly with parallel facets, $F_{p}$ is reduced by more than a factor of 2 compared to perfect spheres. Importantly, visible strong coupling should now be only reached for spacings around $1 \mathrm{~nm}$ (Figure 1-d). On the other hand, because the particle volumes are similar for all geometries, the radiative yields, $\Phi$, computed in Figure 1-c do 
not depend significantly on the particle morphology nor on their relative orientation. Overall, the faceted shape of polycrystalline gold particles will introduce a strong dispersion of emitter-resonator coupling strengths $g$ and lower the probability of experimentally observing strong coupling.

In practice, these theoretical investigations demonstrate that reaching sub-2 $\mathrm{nm}$ spacings in AuNP dimers is essential to observe strong coupling with fluorescent molecules that are introduced in the interparticle gap. Furthermore, they show that $40 \mathrm{~nm}$ AuNPs should be favored but that photoluminescence will then be weak and that scattering spectroscopy is better suited to characterize the optical properties of such hybrid nanostructures. Finally, the emitter-resonator coupling strength remains large even when the transverse position of the emitter is tuned by $\pm 3.5 \mathrm{~nm}$ (Figure S1-b), indicating that several molecules can be coupled simultaneously with the resonator.

\section{Reaching sub-2 nm interparticle spacings reproducibly}

Generally, DNA-templated plasmonic dimers are produced with spacings ranging between $10 \mathrm{~nm}$ and $20 \mathrm{~nm}$ in order to have a nanoscale control over the interparticle distance and over the position of a fluorescent emitter in the gap. ${ }^{19,33-40}$ However, smaller spacings can be reached, in a controlled way, by assembling AuNPs with a DNA strand perpendicular to the dimer axis. ${ }^{49,58}$ Furthermore, the interparticle distance can be actively and progressively reduced by screening the repulsive electrostatic interaction between the negatively charged AuNPs ${ }^{59,60}$ or by a thermal treatment. ${ }^{61,62}$ We combine these strategies by assembling polycrystalline $40 \mathrm{~nm}$ AuNP dimers using a 50bp-long DNA double-strand and studying their plasmon resonance while actively increasing the local ionic strength (Figure 2-a). In practice, one AuNP is functionalized with a 50 bases long DNA single-strand featuring a trithiol group on its 5'-end, while the other is functionalized with the complementary strand, for which the trithiol moiety is on the 3'-end. Hybridization of the DNA strand then drives the self-assembly of the dimer. ${ }^{49}$ 
In order to exploit the DNA double-strand as a scaffold on which a controlled number of emitters can be attached, it is essential to favor the assembly of dimers linked by a single DNA double-helix and to minimize the number of other single-strands attached on the particle surface. This is generally done by minimizing the amount of DNA strands functionalized on the surface of the particles with which dimers can self-assemble. ${ }^{37,59}$ However, because DNA strands are negatively charged like the gold nanoparticles, it is necessary to introduce an excess of trithiolated DNA strands and charge-screening $\mathrm{Na}^{+}$cations to achieve this. ${ }^{37,59,60}$ In that case, the DNA/AuNP ratio is larger than 10 and demonstrating that only one DNA double-strand binds the dimer is generally performed indirectly by introducing a fluorescent molecule on the DNA scaffold and observing photobleaching or photon antibunching. ${ }^{37}$

Alternatively, it is possible to functionalize gold nanoparticles with thiolated DNA singlestrands by lowering the $\mathrm{pH}$ in order to neutralize the phosphate backbone of the DNA. ${ }^{63,64}$ Using this strategy, we can assemble $40 \mathrm{~nm}$ AuNP dimers with a DNA/AuNP ratio as low as 1.25 , clearly evidencing that there is only one DNA double-strand that generally links the particles of each dimer. After electrophoretic purification (Figure S2-a), both methods provide similar results for the assembly of AuNP dimers but the DNA/AuNP ratio is ten times lower in the case of the low-pH approach. We therefore use this technique to assemble $40 \mathrm{~nm}$ AuNP dimers with a DNA/AuNP ratio lower than 2.5. In the obtained hybrid nanostructures observed in electron microscopy (Figure S2-b), the polycrystalline gold particles feature rounded tips and 5-10 nm long facets, in good agreement with the rounded icosahedra considered in the simulations of Figure 1-c-d.

Individual DNA-templated $40 \mathrm{~nm}$ AuNP dimers are then studied by scattering spectroscopy in an inverted darkfield microscope, as shown in Figure 2. The dimers are kept in a buffer solution in a homemade microfluidic chamber with one particle, functionalized with biotin, linked to a Neutravidin-coated glass slide (see Figure S3). First measurements are performed at low ionic strengths $(\mathrm{NaCl}$ concentration of $50 \mathrm{mM})$. In this case, the dimers scatter green-yellow light with an average resonance wavelength of $560 \mathrm{~nm}$, indicating in- 
terparticle distances of the order of $10 \mathrm{~nm} .{ }^{59,60}$ This means that the DNA double-strand is probably partially dehybridized because of electrostatic repulsion between particles. ${ }^{49}$ The local ionic strength is then progressively increased to $250 \mathrm{mM}$, and finally, $1 \mathrm{M}$ in order to reduce the interparticle spacings. As shown qualitatively on darkfield images, and quantitatively in the distributions of longitudinal plasmon resonance frequencies for 100 dimers (Figure 2-c), the hybrid nanostructures experience a significant redshift of their resonance wavelengths by about $100 \mathrm{~nm}$.

At intermediate ionic strengths, plasmon resonance spectra exhibit a main redshifted longitudinal mode and a transverse mode that generally appears as a shoulder (middle case in Figure 2-b). The relative intensity of these two peaks under unpolarized white light depends on the orientation of the dimer in the microfluidic chamber. ${ }^{59}$ The distribution of longitudinal plasmon resonances provides an average wavelength of $602 \mathrm{~nm}$, corresponding to interparticle distances around $4 \mathrm{~nm} .{ }^{59}$ Such spacings typically indicate that the surface ligands on the surface of the particles (short thiolated ethylene glycol oligomers) are in contact but are not interpenetrated. ${ }^{59,60}$ It is not possible to observe this change of the interparticle distance when increasing the ionic strength in electron microscopy, even in cryogenic conditions. Indeed, at $250 \mathrm{mM} \mathrm{NaCl}$, the colloidal suspension of self-assembled dimers is not stable and the nanostructures would aggregate on each other. Aggregation between dimers is not observed in the microfluidic chambers used for scattering spectroscopy as the nanostructures are well-separated spatially with one biotin-functionalized gold particle of each dimer linked to the Neutravidin-coated glass slide. ${ }^{59,60}$

At high ionic strengths, the second blueshifted peak is spectrally well-separated (right case in Figure 2-b) but it does not only correspond to the transverse plasmonic mode. Indeed, at short spacings, the transverse mode overlaps with a quadrupolar longitudinal mode ${ }^{65}$ as shown in BEM simulations (see Figure S4). Importantly, at $1 \mathrm{M} \mathrm{NaCl}$, single nanostructure spectra can be readily fitted by two energy-dependent Lorentzian functions in order to estimate the resonance frequencies and linewidths of these two modes. The distribution 
a
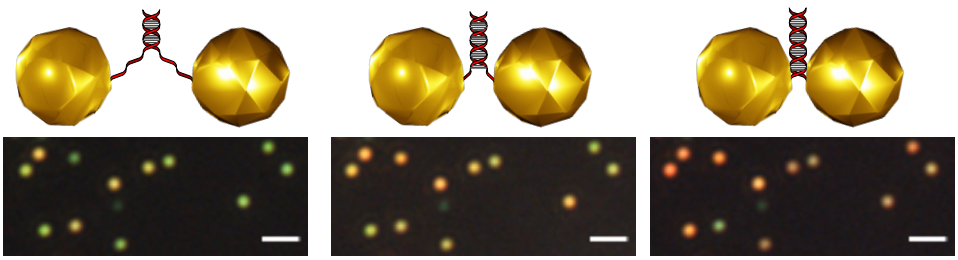

d
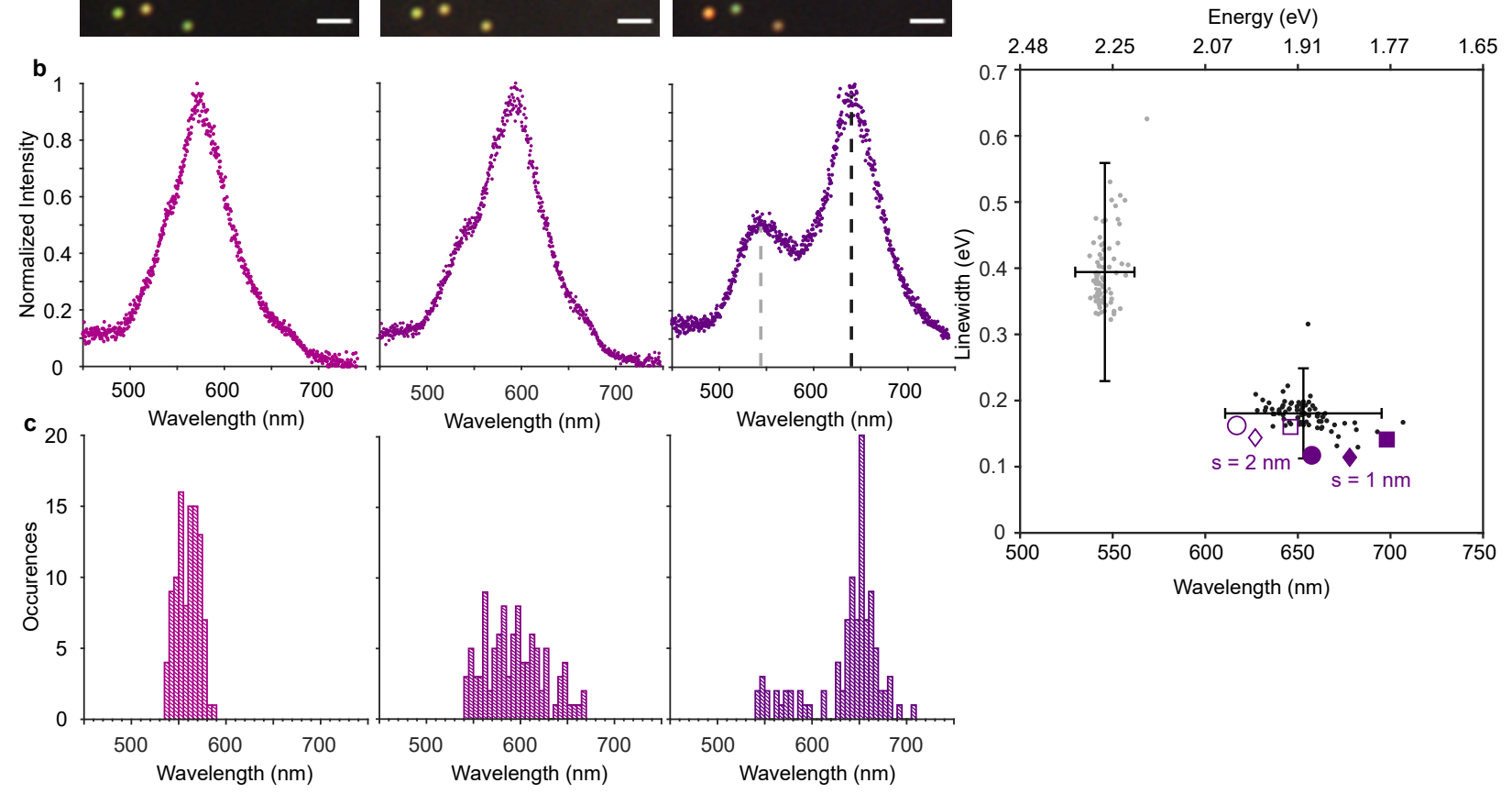

Figure 2: Active reduction of interparticle spacings in DNA-templated $40 \mathrm{~nm}$ AuNP dimers down to sub-2 nm gaps. Schematic representations of $40 \mathrm{~nm}$ diameter AuNP dimers attached by a transverse DNA double-strand and typical darkfield images (a, scale bar is $2 \mu \mathrm{m}$ ), typical single nanostructure resonance spectra (b) and distribution of longitudinal plasmon resonance wavelengths (c) for three different local ionic strengths $(\mathrm{NaCl}$ concentrations from left to right: $50 \mathrm{mM}, 250 \mathrm{mM}$ and $1 \mathrm{M}$ ). (d) Experimental resonance wavelengths and linewidths of the experimental blueshifted (grey points) and redshifted longitudinal modes (black points) measured at $1 \mathrm{M} \mathrm{NaCl}$. The purple symbols correspond to simulated values of the resonance wavelength and linewidth for the longitudinal mode of dimers of $40 \mathrm{~nm}$ AuNPs with an interparticle spacing of $2 \mathrm{~nm}$ (hollow symbols) or $1 \mathrm{~nm}$ (filled symbols): perfect spheres (circles) and rounded icosahedra with a tip facing a planar facet (diamonds) or two parallel facets (squares).

of longitudinal plasmon resonances is separated in two groups with $20 \%$ of dimers having resonances below $620 \mathrm{~nm}$ and $80 \%$ of dimers featuring resonances above $620 \mathrm{~nm}$ (average value of $655 \mathrm{~nm})$. In practice the dimers were left to incubate for $10 \mathrm{~h}$ at $1 \mathrm{M} \mathrm{NaCl}$ to favor the interpenetration of the ligand shells on the particles; ${ }^{59,60}$ which allowed this large spectral redshift for $80 \%$ of the studied dimers. 
To estimate more precisely the interparticle spacings for this majority of AuNP dimers at high ionic strengths, the resonance wavelengths and linewidths of the blueshifted and redshifted plasmonic modes are plotted in Figure 2-d for the $80 \%$ of redshifted dimers and compared to values estimated for the longitudinal mode using BEM simulations for spacings $s$ equal to $1 \mathrm{~nm}$ and $2 \mathrm{~nm}$ and for the three geometries considered in Figures 1-c-d for $40 \mathrm{~nm}$ AuNPs: perfect spheres and faceted particles assembled by two facets or with a tip facing a planar facet. We observe an excellent agreement between the experimental resonance wavelengths and the simulated values for rounded icosahedra when considering spacings ranging between $1 \mathrm{~nm}$ and $2 \mathrm{~nm}$ and the different relative orientations of the two particles. With perfect spheres, more than $30 \%$ of measured dimers feature resonance wavelengths that are red-shifted with respect to the simulated values. Because quantum tunnelling effects tend to blueshift the resonance wavelengths of dimers, ${ }^{51}$ considering spacings below $1 \mathrm{~nm}$ would not provide a better agreement. These measurements thus clearly demonstrate that increasing the ionic strength allows a reproducible and active reduction of the interparticle distance with spacings ranging between $1 \mathrm{~nm}$ and $2 \mathrm{~nm}$ for $80 \%$ of the studied dimers, and that experimental measurements are in better agreement with simulations performed with rounded icosahedra, highlighting the influence of nanoparticle facets.

We also observe that the linewidth of the blueshifted mode is significantly larger than the longitudinal one. This is probably due to the spectral overlap between transverse dipolar and longitudinal quadrupolar modes (Figure S4). Finally, we observe a small discrepancy between the experimental linewidths and the simulated ones. While in all cases, experimental values are larger than the simulated ones, there is a better agreement with calculations performed for rounded icosahedra with two parallel planar facets. Considering the experimental observation that anisotropic particles tend to assemble along larger facets, ${ }^{57}$ this indicates that our assembly scheme probably favors this relative orientation for polycrystalline particles.

The explanation for the larger experimental decay rate of the AuNP dimers compared to 
simulations could be due to the choice of the bulk dielectric constant of gold. Quantum tunnelling effects ${ }^{50,51}$ may introduce extra damping at such small spacings. Another possibility is that the published data for the refractive index of gold ${ }^{48}$ are obtained on rough metal films, which may lead to a slight underestimation of the imaginary part of the dielectric constant as discussed in the literature. ${ }^{66}$ While this discrepancy in resonator damping rates means that visible strong coupling will be experimentally more challenging to observe than estimated in Figure 1, the main result of this analysis remains that sub-2 nm spacings are reached reproducibly.

\section{Experimental observation of strong coupling with 5 dye molecules}

To introduce 5 dye molecules in the nanoscale gap between $40 \mathrm{~nm}$ AuNPs, we substitute the bare 5'-trithiolated DNA strand used in the experiments of Figure 2 for a 5'-trithiolated DNA strand in which 5 cytosine bases are functionalized by an ATTO647N molecule (see the Methods section). ATTO647N molecules where chosen because the peak of their absorption spectrum matches the average longitudinal resonance wavelength measured in Figure 2-d for $40 \mathrm{~nm}$ AuNP dimers at $1 \mathrm{M} \mathrm{NaCl}$, favoring a maximum coupling strength $g$. The organic dyes are separated by 4 or 5 bases, which means that, for a DNA double-strand at physiological conditions with a width of $2 \mathrm{~nm}$, they should be on opposite sides of the DNA helix and their typical distance should be of the order of $2.5 \mathrm{~nm}$, which is sufficient to avoid coherent coupling between emitters. ${ }^{21,67}$

To verify this, we perform ensemble absorbance and fluorescence spectra of DNA doublestrands featuring one or 5 ATTO647N molecules (see Figure S5). We observe that the absorbance measurements are spectrally similar and that the blueshifted shoulder is only slightly larger with 5 ATTO647N molecules than with one, indicating negligible coherent

coupling. ${ }^{67}$ However, the absorbance with 5 ATTO647N molecules is typically 4 times larger than with one molecule, evidencing an imperfect functionalization of amino-modified cytosine bases by ATTO647N molecules. This means that only a fraction of DNA strands will exhibit 
the 5 ATTO647N molecules, while others will only feature 3 or 4 emitters. The fluorescence data are spectrally identical and similar in intensity with one or 5 dye molecules. This demonstrates that, while coherent coupling between emitters is avoided when emitters are separated by $\sim 2.5 \mathrm{~nm}$, there is incoherent dipole-dipole coupling (Förster Resonant Energy Transfer between identical molecules, sometimes called homo-FRET), ${ }^{67}$ which leads to a reduction of the fluorescence quantum yield by a factor of $\sim 4$.

DNA-templated $40 \mathrm{~nm}$ AuNP dimers featuring 5 ATTO647N molecules are analyzed by single-nanostructure scattering spectroscopy similarly to Figure 2. Samples are initially prepared at a moderate $50 \mathrm{mM} \mathrm{NaCl}$ concentration before the ionic strength is progressively increased to $250 \mathrm{mM} \mathrm{NaCl}$ in order to favor the reduction of the interparticle distance while maintaining the DNA double-strand in the gap. A first series of scattering spectra are measured on several hundred isolated dimers before the $\mathrm{NaCl}$ concentration is increased to $1 \mathrm{M}$ and the sample is left to incubate for $10 \mathrm{~h}$ in order to favor the interpenetration of the ligand shells on the AuNPs. ${ }^{59,60}$

As shown in Figure 3-a, we observe, in 3\% of studied dimers, a splitting of the longitudinal mode at $1 \mathrm{M} \mathrm{NaCl}$, which is not visible at $250 \mathrm{mM} \mathrm{NaCl}$ when the interparticle spacing is of the order of $4 \mathrm{~nm}$. It is important to stress that single nanostructure scattering spectra are measured on the same dimers at $250 \mathrm{mM}$ and $1 \mathrm{M} \mathrm{NaCl}$, as exemplified in Figures 2-b and 3-a. This ensures that the hybrid modes, observed when the interparticle distance becomes lower than $2 \mathrm{~nm}$, correspond to a gold nanoparticle dimer featuring a single longitudinal plasmonic mode at $250 \mathrm{mM} \mathrm{NaCl}$. Therefore, the appearance of two redshifted plasmonic modes at short interparticle spacings cannot be attributed to a small aggregate or to a dimer featuring a strongly anisotropic gold particle. Furthermore, the measured energy splittings are never observed in the sample studied in Figure 2 with a bare DNA double-strand. To analyze these coupled eigenmodes more quantitatively, we fit the measured spectra with 3 Lorentzian functions to take into account the blueshifted mode already visible in Figure 2. Since this blueshifted mode has a narrow distribution of resonance energies at $2.27 \pm 0.04$ 

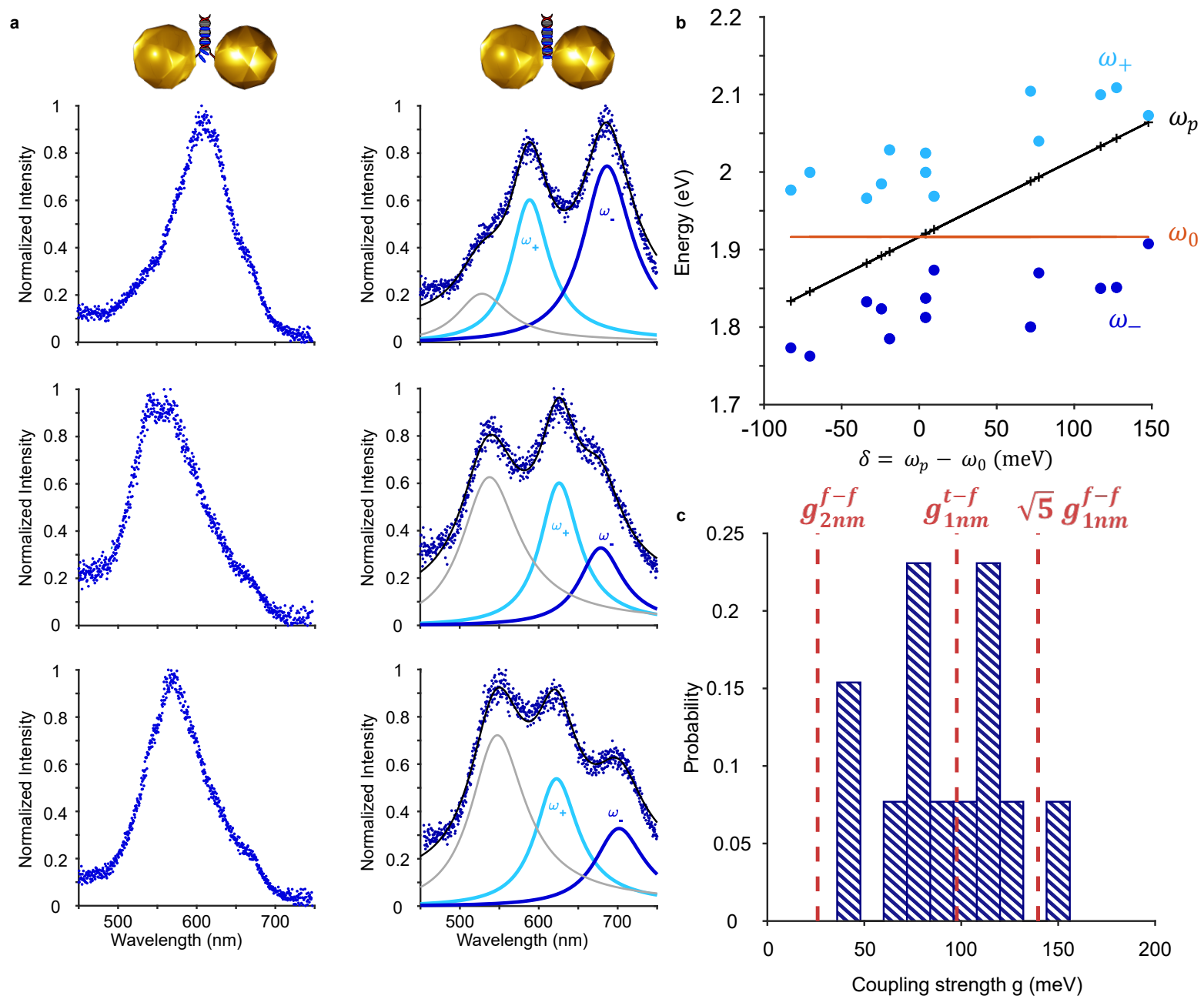

Figure 3: Visible few molecule strong coupling in DNA-templated $40 \mathrm{~nm}$ AuNP dimers at high ionic strengths. (a) Examples of scattering spectra, measured on the same $40 \mathrm{~nm}$ AuNP dimer featuring 5 ATTO647N molecules at intermediate $(250 \mathrm{mM}$, left $)$ and high (1 $\mathrm{M}$, right) $\mathrm{NaCl}$ concentrations. At $1 \mathrm{M} \mathrm{NaCl}$, the spectra are fitted with 3 energy-dependent Lorentzian functions: the blue lines correspond to hybridized eigenmodes $\omega_{ \pm}$while the thin grey line is the blueshifted mode already observed in Figure 2. (b) Distribution of hybrid wavelengths $\omega_{ \pm}$as a function of the energy detuning $\delta=\omega_{p}-\omega_{0}$. The resonance energy of the emitter is plotted as a red line $\left(\omega_{0}\right)$, while the estimated longitudinal plasmon resonance $\left(\omega_{p}\right)$ is shown as black data points and a black line. (c) Distribution of estimated coupling energies $g$, compared to simulated values by BEM for faceted AuNP dimers: rounded icosahedra assembled along parallel facets with interparticle spacings of $1 \mathrm{~nm}\left(g_{1 n m}^{f-f}\right)$ and $2 \mathrm{~nm}\left(g_{2 n m}^{f-f}\right)$; or assembled with a tip facing a planar facet with $s=1 \mathrm{~nm}\left(g_{1 n m}^{t-f}\right)$.

$\mathrm{eV}$, we perform the fitting procedure in Figure 3-a by forcing one resonance energy to be within this range.

The two redshifted hybrid modes $\omega_{ \pm}$are analyzed by considering the longitudinal mode of 
the resonator and the emitter as classical damped harmonic oscillators in order to recover the anticrossing behavior expected in a Jaynes-Cummings model: ${ }^{22,25,44}$

$$
\begin{aligned}
& \omega_{ \pm}=\frac{\omega_{p}+\omega_{0}}{2} \pm \sqrt{g^{2}+\frac{\delta^{2}}{4}}, \\
& \gamma_{ \pm}=\frac{\left(\gamma_{0}-\Gamma_{0}\right)+\gamma_{p}}{2}
\end{aligned}
$$

with $\delta=\omega_{p}-\omega_{0}$ the energy detuning between the resonances of the emitter and of the resonator and $\gamma_{ \pm}$the damping rate of the two hybrid modes. The Lorentzian fitting procedure to determine $\omega_{ \pm}$is therefore validated only when the two hybrid modes feature the same damping rate within a $10 \%$ margin. These experimental estimations of $\omega_{ \pm}$, associated with the tabulated fixed resonance energy of ATTO647N molecules $\left(\omega_{0}=1.916 \mathrm{eV}\right)$, allow us to infer $\omega_{p}$ for each dimer featuring hybrid redshifted modes, as plotted in Figure 3-b. These results nicely evidence the anticrossing behavior expected in a visible strong coupling regime. The estimated values of $\omega_{p}$ provide the energy detuning $\delta$ for each considered resonator and it is then possible to extract the distribution of coupling energies $g$ as shown in Figure 3-c. We compare this distribution with simulated values of $g$. Since experimental scattering data are in better agreement with simulations performed with rounded icosahedra assembled along parallel facets, we consider this orientation with 1 or 5 dye molecules: one longitudinallycoupled emitter with a $2 \mathrm{~nm}$ interparticle spacing $\left(g_{2 n m}^{f-f}\right)$ or 5 identically coupled emitters for a $1 \mathrm{~nm}$ spacing $\left(\sqrt{5} \times g_{1 n m}^{f-f}\right)$. We also consider the possibility that the dimers assemble with a tip facing a planar facet for a $1 \mathrm{~nm}$ spacing $\left(g_{1 n m}^{t-f}\right)$. We observe that the measured coupling strengths agree nicely with classical electrodynamic simulations since the maximum observed value of $g$ is similar to $\sqrt{5} \times g_{1 n m}^{f-f}$ and that the minimum value is of the order of $g_{2 n m}^{f-f}$. In this case (right center graph of Figure $3-\mathrm{a}$ ), the visible strong coupling criterion is not perfectly fulfilled as the $\omega_{-}$hybrid mode is a shoulder of the $\omega_{+}$resonance.

To further ensure that the hybrid eigenmodes observed in Figure 3 can be non-ambiguously attributed to strong coupling between the longitudinal mode of the plasmonic dimer and the 
dye molecules introduced on the DNA strand, we show on Figure 4 the evolution of the scattering spectra of self-assembled dimers after excitation during $10 \mathrm{~s}$ with a picosecond pulsed laser at $635 \mathrm{~nm}$, close to the absorption resonance of ATTO647N. We observe the disappearance of the redshifted hybrid modes and the recovery of a single longitudinal plasmonic mode. The values of $\omega_{p}$ in the strongly-coupled dimers of Figure 4-a are estimated using the coupled-oscillator model used in Figure 3 and reported as a dashed line. We observe that the inferred values of $\omega_{p}$ are a perfect spectral match with the single peaks measured after photoinduced bleaching in Figure 4-b. These experiments therefore demonstrate that the strong coupling regime breaks down once the dye molecules are bleached.

The measurements described in Figures 3 and 4 therefore evidence how introducing 5 ATTO647N emitters in dimers of $40 \mathrm{~nm}$ AuNPs, and then actively decreasing the interparticle spacing below $2 \mathrm{~nm}$, allows the observation of few-molecule strong coupling in single-nanostructure scattering spectroscopy. The measured coupling strengths and the low probability of observing this effect are in excellent agreement with the theoretical investigations of Figure 1. It is however difficult to conclude whether the measured energy splittings are associated with 4 or 5 molecules correctly oriented in a resonator assembled along planar facets; or with 1 of the ATTO647N molecules having, by chance, a correct orientation and position with respect to a sharp tip of one polycrystalline particle in the resonator. To test these hypotheses, it would be interesting to assemble similar DNA-templated dimers with chemically etched spherical gold nanoparticles, which are monocrystalline and do not feature planar facets, ${ }^{54,55,65}$ to experimentally verify whether the probability in observing strong coupling is larger or lower than with polycrystalline particles. Furthermore, we performed experiments using a DNA strand featuring only one ATTO647N molecule, the one closest to the trithiol moiety on the DNA strand, and did not observe hybrid coupled modes. Importantly, at $1 \mathrm{M} \mathrm{NaCl}$ and in the sterically confined gap between the particles (due to the surface ligands in particular), it is difficult to speculate how the DNA and dye molecules are spatially organized. 


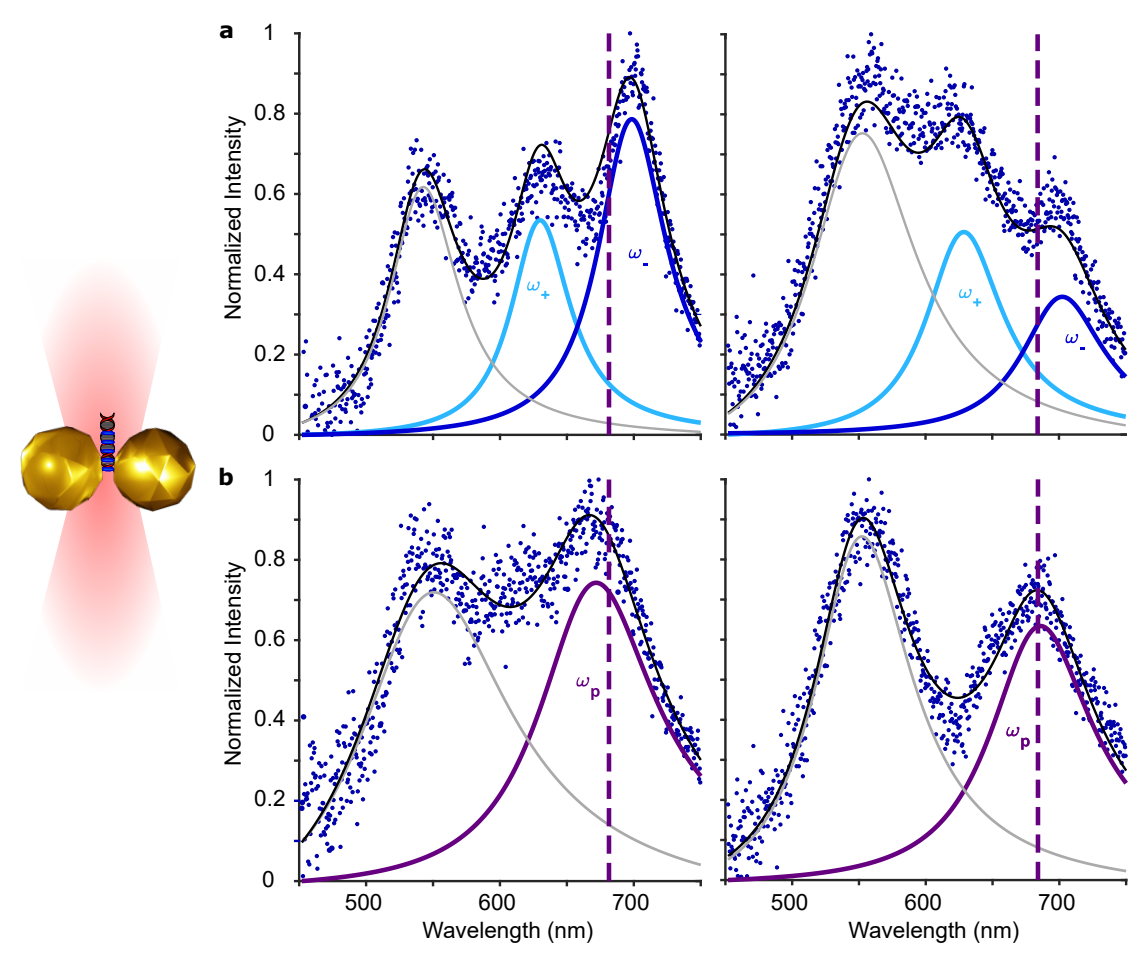

Figure 4: Disappearance of hybrid strongly-coupled modes after photoinduced bleaching of the dye molecules. Scattering spectra of single strongly coupled dimers (blue data) before (a) and after (b) photobleaching of the ATTO647N dye molecules using a pulsed excitation at $635 \mathrm{~nm}$ for $10 \mathrm{~s}$. A Lorentzian fit of these spectra (solid gray and blue lines) allows the estimation of the hybrid eigenenergies $\omega_{ \pm}$and of $\omega_{p}$, which is plotted as a dashed purple line and compared to the Lorentzian fit of the spectrum after bleaching (gray and purple solid lines).

\section{Conclusion}

Observing a visible strong coupling regime with a limited number of quantum emitters is difficult in dimers of gold nanoparticles as these plasmonic resonators feature fast damping rates. We demonstrate here, theoretically and experimentally, that this regime is accessible when sub-2 $\mathrm{nm}$ interparticle spacings are reached in a controlled way. Single nanostructure scattering spectroscopy proves that a DNA-based self-assembly strategy, combined with an increase of the ionic strength, allows us to produce reproducibly $40 \mathrm{~nm}$ AuNP dimers with a sub-2 $\mathrm{nm}$ spacing in proximity to a DNA double-strand on which fluorescent molecules can be readily attached. This allows us to introduce 5 ATTO647N molecules in the nanoscale 
gap and to observe a visible strong coupling regime with experimentally measured coupling strengths that are in excellent agreement with electrodynamic simulations. However, only $3 \%$ of studied dimers feature hybrid coupled modes, probably because of an uncontrolled orientation of the emitters' transition dipoles with respect to the dimer axis, and to the preferential positioning of emitters on facets of gold nanoparticles, which reduces the coupling strength compared to perfect spheres and to emitters positioned on the tips of faceted AuNPs. Interestingly, DNA-based nanotechnology provides a flexible experimental toolbox to overcome these nanofabrication issues: by controlling the orientation of the transition dipoles of dye molecules, ${ }^{68}$ by organizing anisotropic particles with sharp tips with an excellent control over their relative orientation ${ }^{69-71}$ or by functionalizing these sharp tips specifically. ${ }^{72}$ Such hybrid nanostructures are therefore excellent candidates to reach reproducible few- or single-molecule strong coupling at room temperature, in order to exploit the expected optical properties of these nanophotonic systems such as single-photon nonlinearities and ultrafast switching. ${ }^{4,5,7,8}$

\section{Methods}

\section{Synthesis of DNA-templated $40 \mathrm{~nm}$ AuNP dimers}

Commercial $40 \mathrm{~nm}$ diameter AuNPs (BBI, UK) are coated with a negatively charged phosphine ligand (bis-sulfonatophenyl-phenyl-phosphine / BSPP, Strem Chemicals, USA) then rinsed and concentrated by centrifugation following published procedures. ${ }^{49}$ Three different trithiolated 50 bases long DNA strands (Fidelity Systems, USA) are used to functionalize the AuNPs. The same 5' sequence is used either with 5 ATTO647N molecules or without them, while the third strand features the complementary sequence but with a 3' trithiol moiety. The 5 ATTO647N-modified 50 bases long DNA sequence is the following: 5'-trithioGCAXGAAAXCTGGAXACCCXTAAGXAACTCCGTATCAGATGGGAACAGCA-3' ( X $=$ ATTO647N on C base). Dye functionalization of the DNA strand is performed commer- 
cially on amino-modified cytosine bases using N-hydroxysuccinimide ester-modified ATTO647N. Two procedures are then used to obtain DNA-functionalized AuNPs :

- The first one is based on published procedures ${ }^{59}$ and uses $\mathrm{Na}^{+}$ions to screen electrostatic repulsion between DNA strands and AuNPs. In practice, 0.04 pmol of 40 nm BSPP-passivated AuNPs are incubated overnight with varying amounts of 5'- or 3'-trithiolated 50 bases long DNA strands in a $25 \mathrm{mM} \mathrm{NaCl}, 1.17 \mathrm{mM}$ BSPP solution with a final volume of $10 \mu \mathrm{L}$.

- The second one uses an incubation at low-pH to neutralize the negative charges on the DNA strand and favor its interaction with AuNPs. ${ }^{63,64} 0.04 \mathrm{pmol}$ of $40 \mathrm{~nm}$ BSPPpassivated AuNPs are incubated with varying amounts of 5'- or 3'-trithiolated 50 bases long DNA strands in a $1.17 \mathrm{mM}$ BSPP solution with a final volume of $10 \mu \mathrm{L}$. A citrate buffer solution $(\mathrm{pH}=3)$ is then added to reach a final concentration of $20 \mathrm{mM}$. After a 5 min incubation, a HEPES buffer solution $(\mathrm{pH}=7.6)$ is added to reach a final concentration of $100 \mathrm{mM}$. The suspension is then left to incubate overnight before being rinsed by centrifugation in a $0.5 \times$ tris-borate EDTA (TBE) buffer.

In both protocols, 4 samples are prepared with varying DNA/AuNP ratios for the 5'trithiolated strand. For the $\mathrm{NaCl}$ protocol, the different ratios are $12.5 / 25 / 50$ and 100. For the low-pH method, these ratios are reduced by a factor of 10 to $1.25 / 2.5 / 5$ and 10. The 3' trithiolated strand is added in an excess of 3 compared to the 5 ' strand as it is never functionalized with dye molecules and, for this sequence, it is not necessary to favor the attachment of only one DNA strand. ${ }^{59}$

After the overnight incubation, the DNA-conjugated $40 \mathrm{~nm}$ AuNPs are incubated with a $10^{6} \times$ excess of thiolated/methyl-ether terminated ethylene glycol hexamer (Polypure, Norway) for $30 \mathrm{~min}$. These ligands are used to passivate the AuNP surface. The particles featuring the 3'-trithiolated complementary DNA single-strand are incubated with an 8:1 mix of the same ligand and a biotinylated/thiolated ethylene glycol octamer (Polypure, Nor- 
way: the ligand is obtained as a disulfide, which is cleaved by reacting with an excess of BSPP). ${ }^{49}$ The samples are then purified by electrophoresis in a $1 \%$ weight agarose gel, using a $0.5 \times$ TBE running buffer, to remove unbound ligands and DNA strands. The passivated $40 \mathrm{~nm}$ AuNPs, functionalized with either 5'- or 3'-trithiolated DNA strands, are cut from the gel and concentrated by centrifugation before being incubated overnight in stoichiometric amounts in $25 \mathrm{mM} \mathrm{NaCl}$, in order to drive the self-assembly of dimers by hybridization of the complementary DNA strands. The obtained suspensions are once again purified by gel electrophoresis (1\% weight, Figure S2-a). The second slowest band, which corresponds to DNA-templated $40 \mathrm{~nm}$ AuNP dimers (as verified in electron microscopy, see Figure S2-b), is cut from the gel and the recovered suspension is concentrated by centrifugation.

\section{Optical Measurements}

Homemade microfluidic chambers are prepared using published procedures. ${ }^{49}$ In brief, freshly cleaned glass coverslips, which are stacked together with two layers of melted parafilm, are functionalized with BSA-biotin (Sigma-Aldrich, USA) and Neutravidin (Thermo-Scientific, USA) before introducing the dimer suspension at a sub-nanomolar concentration (15 min incubation) and rinsing the chamber with $200 \mu \mathrm{L}$ of a $5 \mathrm{mM}$ Tris, $50 \mathrm{mM} \mathrm{NaCl}$ buffer solution $(\mathrm{pH}=8)$. The $\mathrm{NaCl}$ concentration in the chamber is modified to $250 \mathrm{mM}$ or $1 \mathrm{M}$ by rinsing the chamber with $200 \mu \mathrm{L}$ of a $5 \mathrm{mM}$ Tris, $250 \mathrm{mM}$ or $1 \mathrm{M} \mathrm{NaCl}$ solution. After increasing the ionic strength to $250 \mathrm{mM} \mathrm{NaCl}$, the sample is left to incubate for $2 \mathrm{~h}$ before optical measurements; while, after reaching $1 \mathrm{M} \mathrm{NaCl}$, the sample is left to incubate overnight ( $10 \mathrm{~h})$.

Darkfield images and spectra are measured in an inverted microscope (IX71, Olympus) coupled to a color CCD (Quicam, Roper) and to a fiber-coupled (50 $\mathrm{m}$ core diameter) imaging spectrometer (Acton SP300 with Pixis 100 CCD detector, Princeton Instruments). White light from a $100 \mathrm{~W}$ halogen lamp is focused on the sample using an oil-immersion 1.2-1.4 NA dark-field condenser. Scattered light is collected with a $100 \times 0.6$ NA immersion oil 
objective. Color images of the same chamber, obtained with a $900 \mathrm{~ms}$ acquisition time at 50 mM, $250 \mathrm{mM}$ and $1 \mathrm{M} \mathrm{NaCl}$ concentrations, are given in Figures 2 and S3. These large-scale images allow an estimation of the purity of dimers compared to left-over single particles and larger aggregates. ${ }^{59}$ Indeed, the optical response of single particles is weaker than dimers and, importantly, does not redshift when the $\mathrm{NaCl}$ concentration is increased. Single particles correspond to $\sim 28 \%$ of scattering objects in the sample. Several scatterers with large interaction cross-sections account for less than $2 \%$ of the sample, and are attributed to small aggregates that are not considered in our analysis. Overall, the dimer purity is of the order of $70 \%$ in Figure S3.

Raw single-nanostructure scattering spectra are measured with a 4 s acquisition time. A background spectrum is measured with the same acquisition time on an empty area of the sample. The background is subtracted to the measured spectra before the obtained data are divided by a smoothed background spectrum (from which a constant offset of the CCD detector is subtracted) in order to correct for the wavelength-dependent illumination and detection. ${ }^{59}$

Photoinduced bleaching experiments are performed by exciting for $10 \mathrm{~s}$ a single dimer, through the same $100 \times 0.6$ NA immersion oil objective, with a tunable laser source (iChrome, Toptica) that delivers $3.5 \mathrm{ps}$ pulses at a $40 \mathrm{MHz}$ repetition rate, with a wavelength fixed at $635 \mathrm{~nm}$ and associated with a bandpass filter (Z633/10x, Chroma) for an excitation power ranging between 1 and $2.5 \mathrm{~kW} / \mathrm{cm}^{2}$.

\section{Simulations}

Mie theory calculations are performed using an in-house code. ${ }^{46}$ Boundary Element Method simulations are performed using the MNPBEM toolbox. ${ }^{47}$ The $3 \mathrm{D}$ meshing of the rounded icosahedron is generated using COMSOL and used in BEM simulations. The dielectric constant of gold is taken from tabulated data, ${ }^{48}$ while the refractive index of the environment is chosen at 1.4 to be compatible with experiments performed on DNA-templated gold particle 
dimers. ${ }^{19,37,49}$ We neglect the slight modification of the refractive index of the surrounding buffer solution when the ionic strength is increased $\left(\Delta n \approx 10^{-2}\right)$ as it will introduce a negligible modification of the resonance wavelength of the self-assembled dimers. ${ }^{59}$ In all cases, the total dissipated and radiated powers of a classical electric dipole in the resonator are compared to the power radiated without the resonator in order to compute $F_{p}$ and $\Phi$ for a given position, orientation and emission wavelength. By tuning the emission wavelength for a longitudinally coupled emitter, it is possible to plot $F_{p}$ as a function of energy and infer the resonance wavelength $\omega_{p}$ and decay rate $\gamma_{p}$ of the longitudinal mode of the dimer.

In Figure S4, the scattering spectra of AuNP dimers or AgNCs are computed in BEM by exciting the nanostructure with a plane wave, polarized longitudinally or transversely in the case of dimers.

\section{Acknowledgement}

The authors thank Xiangzhen $\mathrm{Xu}$ for transmission electron microscopy measurements. This work was supported by Agence Nationale de la Recherche via project ANR-15-CE09-0003 and by LABEX WIFI (Laboratory of Excellence within the French Program "Investments for the Future") under references ANR-10-LABX-24 and ANR-10-IDEX-0001-02 PSL*.

\section{Supporting Information Available}

The Supporting Information is available free of charge on the ACS Publications website. Complementary figures describing the purification of nanoparticle dimers, simulations of strong coupling figure of merits \& scattering spectra, and absorbance \& fluorescence spectra of dye-conjugated DNA strands. 


\section{References}

1. Zumofen, G.; Mojarad, N. M.; Sandoghdar, V.; Agio, M. Perfect Reflection of Light by an Oscillating Dipole. Phys. Rev. Lett. 2008, 101, 180404.

2. Wrigge, G.; Gerhardt, I.; Hwang, J.; Zumofen, G.; Sandoghdar, V. Efficient Coupling of Photons to a Single Molecule and the Observation of its Resonance Fluorescence. Nat. Phys. 2008, 4, 60-66.

3. Moerner, W. E.; Orrit, M. Illuminating Single Molecules in Condensed Matter. Science 1999, 283, 1670-1676.

4. Birnbaum, K. M.; Boca, A.; Miller, R.; Boozer, A. D.; Northup, T. E.; Kimble, H. J. Photon Blockade in an Optical Cavity with One Trapped Atom. Nature 2005, 436, $87-90$.

5. Chang, D. E.; Sørensen, A. S.; Demler, E. A.; Lukin, M. D. A Single-Photon Transistor Using Nano-Scale Surface Plasmons. Nat. Phys. 2007, 807-812.

6. Hwang, J.; Pototschnig, M.; Lettow, R.; Zumofen, G.; Renn, A.; Götzinger, S.; Sandoghdar, V. A Single-Molecule Optical Transistor. Nature 2009, 460, 76-80.

7. Loo, V.; Arnold, C.; Gazzano, O.; Lemaître, A.; Sagnes, I.; Krebs, O.; Voisin, P.; Senellart, P.; Lanco, L. Optical Nonlinearity for Few-Photon Pulses on a Quantum Dot-Pillar Cavity Device. Phys. Rev. Lett. 2012, 109, 166806.

8. Volz, T.; Reinhard, A.; Winger, M.; Badolato, A.; Hennessy, K. J.; Hu, E. L.; Imamoğlu, A. Ultrafast All-Optical Switching by Single Photons. Nat. Photon. 2012, 6, 605-609.

9. Törmä, P.; Barnes, W. L. Strong Coupling between Surface Plasmon Polaritons and Emitters: A Review. Rep. Prog. Phys. 2014, 78, 013901. 
10. Baranov, D. G.; Wersäll, M.; Cuadra, J.; Antosiewicz, T. J.; Shegai, T. Novel Nanostructures and Materials for Strong Light-Matter Interactions. ACS Photonics 2018, 5, $24-42$.

11. Pustovit, V. N.; Shahbazyan, T. V. Plasmon-Mediated Superradiance near Metal Nanostructures. Phys. Rev. B 2010, 82, 075429.

12. Coles, D. M.; Yang, Y.; Wang, Y.; Grant, R. T.; Taylor, R. A.; Saikin, S. K.; AspuruGuzik, A.; Lidzey, D. G.; Tang, J. K.-H.; Smith, J. M. Strong Coupling between Chlorosomes of Photosynthetic Bacteria and a Confined Optical Cavity Mode. Nat. Commun. 2014, 5, 5561.

13. Coles, D. M.; Somaschi, N.; Michetti, P.; Clark, C.; Lagoudakis, P. G.; Savvidis, P. G.; Lidzey, D. G. Polariton-Mediated Energy Transfer between Organic Dyes in a Strongly Coupled Optical Microcavity. Nat. Mater. 2014, 13, 712-719.

14. Groenhof, G.; Toppari, J. J. Coherent Light Harvesting through Strong Coupling to Confined Light. J. Phys. Chem. Lett. 2018, 9, 4848-4851.

15. Hutchison, J. A.; Schwartz, T.; Genet, C.; Devaux, E.; Ebbesen, T. W. Modifying Chemical Landscapes by Coupling to Vacuum Fields. Angew. Chem. Int. Ed. 2012, 51, $1592-1596$.

16. Galego, J.; Garcia-Vidal, F. J.; Feist, J. Suppressing Photochemical Reactions with Quantized Light Fields. Nat. Commun. 2016, 7, 13841.

17. Thomas, A.; Lethuillier-Karl, L.; Nagarajan, K.; Vergauwe, R. M. A.; George, J.; Chervy, T.; Shalabney, A.; Devaux, E.; Genet, C.; Moran, J.; Ebbessen, T. W. Tilting a Ground-State Reactivity Landscape by Vibrational Strong Coupling. Science 2019, 363, 615-619. 
18. Yoshie, T.; Scherer, A.; Hendrickson, J.; Khitrova, G.; Gibbs, H. M.; Rupper, G.; Ell, C.; Shchekin, O. B.; Deppe, D. G. Vacuum Rabi Splitting with a Single Quantum Dot in a Photonic Crystal Nanocavity. Nature 2004, 432, 200-203.

19. Busson, M. P.; Rolly, B.; Stout, B.; Bonod, N.; Bidault, S. Accelerated Single Photon Emission from Dye Molecule-Driven Nanoantennas Assembled on DNA. Nat. Commun. 2012, 3, 962 .

20. Bellessa, J.; Bonnand, C.; Plenet, J. C.; Mugnier, J. Strong Coupling between Surface Plasmons and Excitons in an Organic Semiconductor. Phys. Rev. Lett. 2004, 93, 036404.

21. Delga, A.; Feist, J.; Bravo-Abad, J.; Garcia-Vidal, F. J. Theory of Strong Coupling between Quantum Emitters and Localized Surface Plasmons. J. Opt. 2014, 16, 114018.

22. Zengin, G.; Wersäll, M.; Nilsson, S.; Antosiewicz, T. J.; Käll, M.; Shegai, T. Realizing Strong Light-Matter Interactions between Single-Nanoparticle Plasmons and Molecular Excitons at Ambient Conditions. Phys. Rev. Lett. 2015, 114, 157401.

23. Roller, E.-M.; Argyropoulos, C.; Högele, A.; Liedl, T.; Pilo-Pais, M. Plasmon-Exciton Coupling Using DNA Templates. Nano Lett. 2016, 16, 5962-5966.

24. Pelton, M.; Storm, S. D.; Leng, H. Strong Coupling of Emitters to Single Plasmonic Nanoparticles: Exciton-Induced Transparency and Rabi Splitting. Nanoscale 2019, 11, $14540-14552$.

25. Chikkaraddy, R.; Nijs, B. d.; Benz, F.; Barrow, S. J.; Scherman, O. A.; Rosta, E.; Demetriadou, A.; Fox, P.; Hess, O.; Baumberg, J. J. Single-Molecule Strong Coupling at Room Temperature in Plasmonic Nanocavities. Nature 2016, 535, 127-130.

26. Leng, H.; Szychowski, B.; Daniel, M.-C.; Pelton, M. Strong Coupling and Induced Transparency at Room Temperature with Single Quantum Dots and Gap Plasmons. Nat. Commun. 2018, 9, 4012. 
27. Chikkaraddy, R.; Turek, V.; Kongsuwan, N.; Benz, F.; Carnegie, C.; van de Goor, T.; de Nijs, B.; Demetriadou, A.; Hess, O.; Keyser, U. F.; Baumberg, J. J. Mapping Nanoscale Hotspots with Single-Molecule Emitters Assembled into Plasmonic Nanocavities Using DNA Origami. Nano Lett. 2018, 18, 405-411.

28. Santhosh, K.; Bitton, O.; Chuntonov, L.; Haran, G. Vacuum Rabi Splitting in a Plasmonic Cavity at the Single Quantum Emitter Limit. Nat. Commun. 2016, 7, 11823.

29. Groß, H.; Hamm, J. M.; Tufarelli, T.; Hess, O.; Hecht, B. Near-Field Strong Coupling of Single Quantum Dots. Sci. Adv. 2018, 4, eaar4906.

30. Seeman, N. C. DNA in a Material World. Nature 2003, 421, 427-431.

31. Alivisatos, A. P.; Johnsson, K. P.; Peng, X. G.; Wilson, T. E.; Loweth, C. J.; Bruchez, M. P.; Schultz, P. G. Organization of "Nanocrystal Molecules" Using DNA. Nature 1996, 382, 609-611.

32. Elghanian, R.; Storhoff, J. J.; Mucic, R. C.; Letsinger, R. L.; Mirkin, C. A. Selective Colorimetric Detection of Polynucleotides Based on the Distance-Dependent Optical Properties of Gold Nanoparticles. Science 1997, 27\%, 1078-1081.

33. Busson, M. P.; Rolly, B.; Stout, B.; Bonod, N.; Wenger, J.; Bidault, S. Photonic Engineering of Hybrid Metal-Organic Chromophores. Angew. Chem. Int. Ed. 2012, 51, $11083-11087$.

34. Acuna, G. P.; Moller, F. M.; Holzmeister, P.; Beater, S.; Lalkens, B.; Tinnefeld, P. Fluorescence Enhancement at Docking Sites of DNA-Directed Self-Assembled Nanoantennas. Science 2012, 338, 506-510.

35. Busson, M. P.; Bidault, S. Selective Excitation of Single Molecules Coupled to the Bright Mode of a Plasmonic Cavity. Nano Lett. 2014, 14, 284-288. 
36. Puchkova, A.; Vietz, C.; Pibiri, E.; Wünsch, B.; Sanz Paz, M.; Acuna, G. P.; Tinnefeld, P. DNA Origami Nanoantennas with over 5000-fold Fluorescence Enhancement and SingleMolecule Detection at $25 \mu \mathrm{M}$. Nano Lett. 2015, 15, 8354-8359.

37. Bidault, S.; Devilez, A.; Maillard, V.; Lermusiaux, L.; Guigner, J.-M.; Bonod, N.; Wenger, J. Picosecond Lifetimes with High Quantum Yields from Single-PhotonEmitting Colloidal Nanostructures at Room Temperature. ACS Nano 2016, 10, 48064815.

38. Xin, L.; Lu, M.; Both, S.; Pfeiffer, M.; Urban, M. J.; Zhou, C.; Yan, H.; Weiss, T.; Liu, N.; Lindfors, K. Watching a Single Fluorophore Molecule Walk into a Plasmonic Hotspot. ACS Photonics 2019, 6, 985-993.

39. Nicoli, F.; Zhang, T.; Hübner, K.; Jin, B.; Selbach, F.; Acuna, G.; Argyropoulos, C.; Liedl, T.; Pilo-Pais, M. DNA-Mediated Self-Assembly of Plasmonic Antennas with a Single Quantum Dot in the Hot Spot. Small 2019, 15, 1804418.

40. Trofymchuk, K.; Glembockyte, V.; Grabenhorst, L.; Steiner, F.; Vietz, C.; Close, C.; Pfeiffer, M.; Richter, L.; Schütte, M. L.; Selbach, F.; Yaadav, R.; Zähringer, J.; Wei, Q.; Ozcan, A.; Lalkens, B.; Acuna, G. P.; Tinnefeld, P. Addressable Nanoantennas with Cleared Hotspots for Single-Molecule Detection on a Portable Smartphone Microscope. Nat. Commun. 2021, 12, 950.

41. Bidault, S.; Devilez, A.; Ghenuche, P.; Stout, B.; Bonod, N.; Wenger, J. Competition between Forster Resonance Energy Transfer and Donor Photodynamics in Plasmonic Dimer Nanoantennas. ACS Photonics 2016, 3, 895-903.

42. Aissaoui, N.; Moth-Poulsen, K.; Käll, M.; Johansson, P.; Marcus Wilhelmsson, L.; Albinsson, B. FRET Enhancement close to Gold Nanoparticles Positioned in DNA Origami Constructs. Nanoscale 2017, 9, 673-683. 
43. Bohlen, J.; Cuartero-González, A.; Pibiri, E.; Ruhlandt, D.; Fernández-Domínguez, A. I.; Tinnefeld, P.; Acuna, G. P. Plasmon-Assisted Förster Resonance Energy Transfer at the Single-Molecule Level in the Moderate Quenching Regime. Nanoscale 2019, 11, $7674-7681$.

44. Bouchet, D.; Carminati, R. Quantum Dipole Emitters in Structured Environments: A Scattering Approach: Tutorial. J. Opt. Soc. Am. A 2019, 36, 186-195.

45. Bharadwaj, P.; Novotny, L. Spectral Dependence of Single Molecule Fluorescence Enhancement. Opt. Express 2007, 15, 14266-14274.

46. Stout, B.; Devilez, A.; Rolly, B.; Bonod, N. Multipole Methods for Nanoantennas Design: Applications to Yagi-Uda Configurations. J. Opt. Soc. Am. B 2011, 28, 1213-1223.

47. Hohenester, U.; Trügler, A. MNPBEM - A Matlab Toolbox for the Simulation of Plasmonic Nanoparticles. Comput. Phys. Commun. 2012, 183, 370-381.

48. Johnson, P. B.; Christy, R. W. Optical Constants of the Noble Metals. Phys. Rev. B 1972, 6, 4370-4379.

49. Busson, M. P.; Rolly, B.; Stout, B.; Bonod, N.; Larquet, E.; Polman, A.; Bidault, S. Optical and Topological Characterization of Gold Nanoparticle Dimers Linked by a Single DNA Double Strand. Nano Lett. 2011, 11, 5060-5065.

50. Savage, K. J.; Hawkeye, M. M.; Esteban, R.; Borisov, A. G.; Aizpurua, J.; Baumberg, J. J. Revealing the Quantum Regime in Tunnelling Plasmonics. Nature 2012, 491, $574-577$.

51. Cha, H.; Yoon, J. H.; Yoon, S. Probing Quantum Plasmon Coupling Using Gold Nanoparticle Dimers with Tunable Interparticle Distances Down to the Subnanometer Range. ACS Nano 2014, 8, 8554-8563. 
52. Brinks, D.; Stefani, F. D.; Kulzer, F.; Hildner, R.; Taminiau, T. H.; Avlasevich, Y.; Müllen, K.; van Hulst, N. F. Visualizing and Controlling Vibrational Wave Packets of Single Molecules. Nature 2010, 465, 905-908.

53. Sonnichsen, C.; Reinhard, B. M.; Liphardt, J.; Alivisatos, A. P. A Molecular Ruler Based on Plasmon Coupling of Single Gold and Silver Nanoparticles. Nat. Biotechnol. 2005, 23, 741-745.

54. Huh, J.-H.; Lee, J.; Lee, S. Comparative Study of Plasmonic Resonances between the Roundest and Randomly Faceted Au Nanoparticles-on-Mirror Cavities. ACS Photonics 2018, 5, 413-421.

55. Yoon, J. H.; Selbach, F.; Schumacher, L.; Jose, J.; Schlücker, S. Surface Plasmon Coupling in Dimers of Gold Nanoparticles: Experiment and Theory for Ideal (Spherical) and Nonideal (Faceted) Building Blocks. ACS Photonics 2019, 6, 642-648.

56. Novotny, L.; Bian, R. X.; Xie, X. S. Theory of Nanometric Optical Tweezers. Phys. Rev. Lett. 1997, 79, 645-648.

57. Lermusiaux, L.; Nisar, A.; Funston, A. M. Flexible Synthesis of High-Purity Plasmonic Assemblies. Nano Res. 2021, 14, 635-645.

58. Bidault, S.; de Abajo, F. J. G.; Polman, A. Plasmon-Based Nanolenses Assembled on a Well-Defined DNA Template. J. Am. Chem. Soc. 2008, 130, 2750-2751.

59. Lermusiaux, L.; Maillard, V.; Bidault, S. Widefield Spectral Monitoring of Nanometer Distance Changes in DNA-Templated Plasmon Rulers. ACS Nano 2015, 9, 978-990.

60. Lermusiaux, L.; Bidault, S. Increasing the Morphological Stability of DNA-Templated Nanostructures with Surface Hydrophobicity. Small 2015, 11, 5696-5704.

61. Simoncelli, S.; Roller, E.-M.; Urban, P.; Schreiber, R.; Turberfield, A. J.; Liedl, T.; Lohmüller, T. Quantitative Single-Molecule Surface-Enhanced Raman Scattering by 
Optothermal Tuning of DNA Origami-Assembled Plasmonic Nanoantennas. ACS Nano 2016, 10, 9809-9815.

62. Lermusiaux, L.; Bidault, S. Temperature-Dependent Plasmonic Responses from Gold Nanoparticle Dimers Linked by Double-Stranded DNA. Langmuir 2018, 34 , $14946-14953$.

63. Zhang, X.; Servos, M. R.; Liu, J. Instantaneous and Quantitative Functionalization of Gold Nanoparticles with Thiolated DNA Using a pH-Assisted and Surfactant-Free Route. J. Am. Chem. Soc. 2012, 134, 7266-7269.

64. Schreiber, R.; Do, J.; Roller, E.-M.; Zhang, T.; Schüller, V. J.; Nickels, P. C.; Feldmann, J.; Liedl, T. Hierarchical Assembly of Metal Nanoparticles, Quantum Dots and Organic Dyes Using DNA Origami Scaffolds. Nat. Nanotechnol. 2014, 9, 74-78.

65. Yoon, J. H.; Selbach, F.; Langolf, L.; Schlücker, S. Ideal Dimers of Gold Nanospheres for Precision Plasmonics: Synthesis and Characterization at the Single-Particle Level for Identification of Higher Order Modes. Small 2018, 14, 1702754.

66. Olmon, R. L.; Slovick, B.; Johnson, T. W.; Shelton, D.; Oh, S.-H.; Boreman, G. D.; Raschke, M. B. Optical Dielectric Function of Gold. Phys. Rev. B 2012, 86, 235147.

67. Conroy, E. M.; Li, J. J.; Kim, H.; Algar, W. R. Self-Quenching, Dimerization, and Homo-FRET in Hetero-FRET Assemblies with Quantum Dot Donors and Multiple Dye Acceptors. J. Phys. Chem. C 2016, 120, 17817-17828.

68. Gopinath, A.; Thachuk, C.; Mitskovets, A.; Atwater, H. A.; Kirkpatrick, D.; Rothemund, P. W. K. Absolute and Arbitrary Orientation of Single-Molecule Shapes. Science 2021, 371, eabd6179.

69. Kuzyk, A.; Schreiber, R.; Zhang, H.; Govorov, A. O.; Liedl, T.; Liu, N. Reconfigurable 3D Plasmonic Metamolecules. Nat. Mater. 2014, 13, 862-866. 
70. Zhan, P.; Dutta, P. K.; Wang, P.; Song, G.; Dai, M.; Zhao, S.-X.; Wang, Z.-G.; Yin, P.; Zhang, W.; Ding, B.; Ke, Y. Reconfigurable Three-Dimensional Gold Nanorod Plasmonic Nanostructures Organized on DNA Origami Tripod. ACS Nano 2017, 11, 1172-1179.

71. Lan, X.; Su, Z.; Zhou, Y.; Meyer, T.; Ke, Y.; Wang, Q.; Chiu, W.; Liu, N.; Zou, S.; Yan, H.; Liu, Y. Programmable Supra-Assembly of a DNA Surface Adapter for Tunable Chiral Directional Self-Assembly of Gold Nanorods. Angew. Chem. Int. Ed. 2017, 56, 14632-14636.

72. Niu, R.; Song, C.; Gao, F.; Fang, W.; Jiang, X.; Ren, S.; Zhu, D.; Su, S.; Chao, J.; Chen, S.; Fan, C.; Wang, L. DNA Origami-Based Nanoprinting for the Assembly of Plasmonic Nanostructures with Single-Molecule Surface-Enhanced Raman Scattering. Angew. Chem. Int. Ed. 2021, 60, 11695-11701. 
TOC graphic
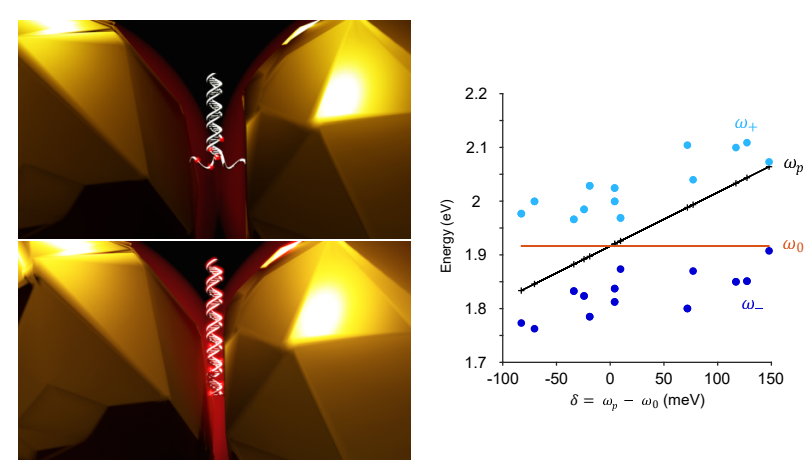